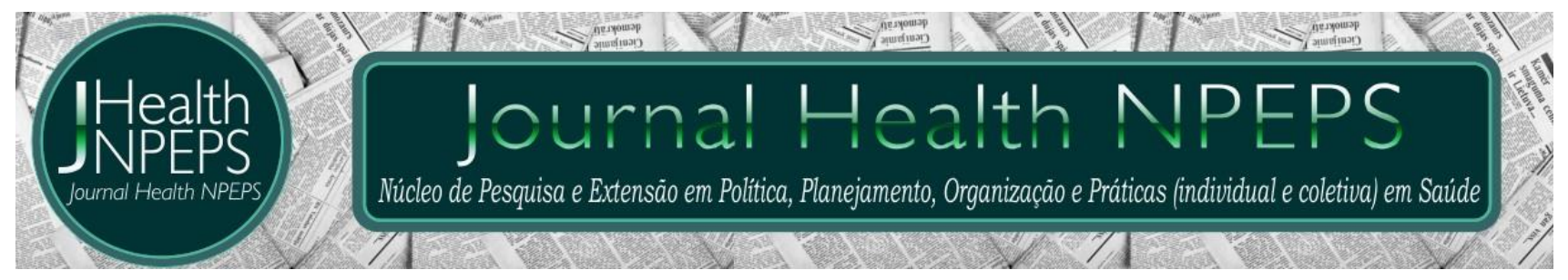

http://dx.doi.org/10.30681/252610104603

ARTIGO ORIGINAL

\title{
Conhecimento de enfermeiros na abordagem à vítima de traumatismo cranioencefálico
}

\section{Knowledge of nurses in the approach to the victim of cranioencephalic traumatism}

\section{Conocimiento de enfermeras en el enfoque de la víctima del traumatismo cranioencefálico}

\section{Fabiana Rezer $^{1}$, Bruno Felipe Oliveira Pereira ${ }^{2}$, Wladimir Rodrigues Faustino ${ }^{3}$}

\begin{abstract}
RESUMO
Objetivo: verificar o conhecimento de enfermeiros sobre o traumatismo cranioencefálico e a Escala de Coma de Glasgow. Método: estudo exploratório e com abordagem quantitativa, realizado entre novembro e dezembro de 2019, a partir de entrevistas com enfermeiros atuantes em serviços hospitalares de urgência e emergência. A coleta de dados ocorreu com aplicação de dois instrumentos. Para análise dos dados, utilizou-se a análise do percentual de concordância. Resultados: $90 \%$ dos enfermeiros já atenderam pacientes com traumatismo cranioencefálico e $95 \%$ referem estar preparados para esse atendimento. Grande parte dos enfermeiros dizem que existem barreiras no atendimento às vítimas de traumatismo crânio encefálico (60\%), como equipe despreparada (20\%) e falta de estrutura (40\%). Em relação a classificação do traumatismo, $70 \%$ acertaram quanto ao leve, $95 \%$ ao moderado e $75 \%$ ao grave. Conclusão: o estudo aponta a importância dos enfermeiros se manterem atualizados no atendimento às vítimas de traumatismo cranioencefálico, além da necessidade de educação permanente e aperfeiçoamento da equipe, para melhoria do atendimento e segurança do paciente.

Descritores: Traumatismos Craniocerebrais; Enfermeiras e Enfermeiros; Cuidados de Enfermagem.
\end{abstract}

\section{ABSTRACT}

Objective: to verify nurses' knowledge about traumatic brain injury and the Glasgow Coma Scale. Method: exploratory study with a quantitative approach, carried out

\footnotetext{
${ }^{1}$ Enfermeira. Mestre. Faculdade do Norte de Mato Grosso. Guarantã do Norte, Mato Grosso, Brasil. E-mail: fabianarezer@hotmail.com ORCID ID: http://orcid.org/0000-0001-8878-1056 Autor para correspondência Endereço: Rua dos oitys, n150, Jardim Vitória, Guarantã do Norte, Mato Grosso, Brasil.

${ }^{2}$ Enfermeiro. Especialista. Faculdade do Norte de Mato Grosso. Guarantã do Norte, Mato Grosso, Brasil. E-mail: brunoofelipe269@hotmail.com ORCID ID: ORCID ID: http://orcid.org/0000-0001-8512-1503

${ }^{3}$ Enfermeiro. Mestre. Faculdade do Norte de Mato Grosso. Guarantã do Norte, Mato Grosso, Brasil. E-mail: faustino cfn@yahoo.com.br ORCID ID: ORCID ID: http://orcid.org/0000-0002-1272-9689
} 
between November and December 2019, based on interviews with nurses working in urgent and emergency hospital services. Data collection occurred with the application of two instruments. For data analysis, analysis of the percentage of agreement. Results: $90 \%$ of nurses have seen patients with traumatic brain injury and $95 \%$ say they are prepared for this service. Most nurses say that there are barriers in caring for victims of traumatic brain injury (60\%), such as unprepared staff (20\%) and lack of structure (40\%). Regarding the classification of trauma, 70\% were right about mild, 95\% moderate and $75 \%$ severe. Conclusion: the study points out the importance of nurses to keep up to date in caring for victims of traumatic brain injury, in addition to the need for permanent education and staff improvement, to improve care and patient safety.

Descriptors: Craniocerebral Trauma; Nurses; Nursing Care.

\section{RESUMEN}

Objetivo: verificar el conocimiento de las enfermeras sobre la lesión cerebral traumática y la Escala de Coma de Glasgow. Método: estudio exploratorio con abordaje cuantitativo, realizado entre noviembre y diciembre de 2019, a partir de entrevistas a enfermeros que laboran en servicios hospitalarios de urgencias y emergencias. La recolección de datos ocurrió con la aplicación de dos instrumentos. Para el análisis de los datos se utilizó el análisis del porcentaje de acuerdo. Resultados: $90 \%$ de los enfermeros ha atendido a pacientes con traumatismo craneoencefálico y el $95 \%$ dice estar preparado para este servicio. La mayoría de los enfermeros dice que existen barreras en el cuidado de las víctimas de una lesión cerebral traumática (60\%), como el personal no preparado (20\%) y la falta de estructura (40\%). En cuanto a la clasificación del trauma, $70 \%$ tenía razón sobre leve, 95\% moderado y $75 \%$ grave. Conclusión: el estudio destaca la importancia de que las enfermeras se mantengan al día en la atención a las víctimas de traumatismo craneoencefálico, además de la necesidad de educación permanente y mejora del personal, para mejorar la atención y seguridad del paciente.

Descriptores: Traumatismos Craneocerebrales; Enfermeras y Enfermeros; Atención de enfermería.

\section{INTRODUÇÃO}

O Traumatismo Cranioencefálico

(TCE) é um conjunto de lesões causadas por fatores externos que acometem o couro cabeludo, crânio e seus componentes internos, sendo capaz de produzir consequências neurológicas agudas e crônicas. É considerado um dos principais traumas notificados nos serviços de urgência e emergência, e está associado a elevados níveis de morbimortalidade ${ }^{0-4}$.

No Brasil, O TCE atinge 500.000 mil pessoas hospitalizadas por ano, com taxas de 14 a 30 óbitos a cada 100.000 mil pessoas. Estima-se que entre 75 a 100 mil pessoas morrem nas primeiras horas do trauma, e outras 70 a 90 mil pessoas ficarão com sequelas irreversíveis. Destes, destacam-se os homens que têm maior risco de se 
envolver em acidentes do que as mulheres ${ }^{5,6}$.

A qualidade do atendimento inicial nesses casos é fundamental para a vítima de TCE, pois intervenções imediatas geram melhores prognósticos. A aplicação da Escala de Coma de Glasgow (ECG) é uma das principais ações nesses atendimentos, pois a determinação do estado neurológico permite a breve instituição terapêutica ${ }^{3-}$ 5 .

Entre os profissionais envolvidos no acolhimento e primeiros cuidados com esse perfil de vítima, destaca-se o enfermeiro, com assistência qualificada em suporte de vida. Essa assistência deve incluir comunicação favorável, imobilização e manutenção da respiração, hemodinâmica e nível de consciência $^{6,7}$, porém não se limita a aspectos clínicos. A liderança, habilidades específicas junto a pacientes graves e/ou com eventos clínicos mais complexos e a coordenação do cuidado seguro, consolidam as atribuições deste profissional junto à vítima com TCE7-Erro! Fonte de referência não encontrada.

Todavia, não há uniformidade nos conhecimentos desses profissionais, pelas particularidades na formação, aprimoramentos individuais e ineficiência de programas de educação permanente nos serviços. Diante disso, o presente estudo objetivou verificar o conhecimento de enfermeiros sobre o TCE e ECG.

\section{MÉTODO}

Trata-se de estudo exploratório e com abordagem quantitativa, baseado no Guidelines for Accurate and Transparent Health Estimates Reporting (GATHER). 0 estudo foi desenvolvido entre novembro e dezembro de 2019, com 22 enfermeiros atuantes nos serviços de urgência e emergência de um município na Região Norte de Mato Grosso. A amostra foi identificada pelo cálculo amostral, com erro amostral de $4 \%$, totalizado a 20 enfermeiros como suficiente.

Teve como critérios de inclusão, enfermeiros de ambos os sexos e que declararam no mínimo com 12 meses de experiência profissional em serviços de urgência e emergência, e excluídos aqueles que estivessem de licença, férias, folga ou atestado.

Os dados foram coletados através de dois questionários, o primeiro foi um questionário semiestruturado, elaborado pelos autores. Este instrumento abordou aspectos sociodemográficos (faixa etária, gênero, 
nível de formação, tempo de trabalho na instituição, tempo de trabalho em serviço de urgência e emergência). 0 segundo questionário foi extraído do estudo de Boratelli10 ${ }^{10}$ questionário contém dados específicos sobre TCE (Se trabalha com TCE, se tem preparo para assistir a vítima, se existe alguma barreira, sobre a escala de Glasgow relacionado aos pontos, seus objetivos, local de utilização e suas dificuldades).

A pesquisa pretendeu responder as seguintes questões norteadoras: Qual o conhecimento dos enfermeiros sobre TCE? Qual a maior dificuldade dos enfermeiros atuantes em serviços de urgência e emergência na identificação dos pacientes com TCE?

As entrevistas foram individuais, realizadas em ambiente privativo nos próprios serviços de saúde, por pesquisador previamente treinado. As entrevistas tiveram duração média de 30 minutos, aplicado no horário que os enfermeiros aguardavam para iniciar o plantão.

A análise dos dados obtidos foi realizada com o software Stattistical Package for Social Sciences (SPSS) versão
19.0 para avaliar frequência absoluta e relativa, sendo apresentados em tabelas. Para análise do percentual, realizou-se o cálculo de concordância, considerado satisfatório acima de $80 \%$.

0 estudo atendeu as diretrizes preconizadas pela Resolução $\mathrm{n}^{\circ} 466$ de 12 de dezembro de 2012 do Comitê de Ética em Pesquisas com Seres Humanos, com CAAE: 13907219.4.0000.8928 e parecer de aprovação n. 3.422.987. Todos os participantes assinaram o Termo de Consentimento Livre e Esclarecido (TCLE).

\section{RESULTADOS}

A amostra foi constituída de 20 participantes, a maioria do sexo feminino $(70 \%)$, grande parte estavam na faixa entre 31 a 40 anos (40\%), com trabalho na instituição entre um e cinco anos (65\%). O tempo de atuação na enfermagem foi entre 11 e 20 anos (40\%) e especialistas (65\%). 
Tabela 1 - Caracterização dos enfermeiros em relação a dados socio demográficos. Novembro a dezembro de 2019. Região Norte de Mato Grosso, Brasil.

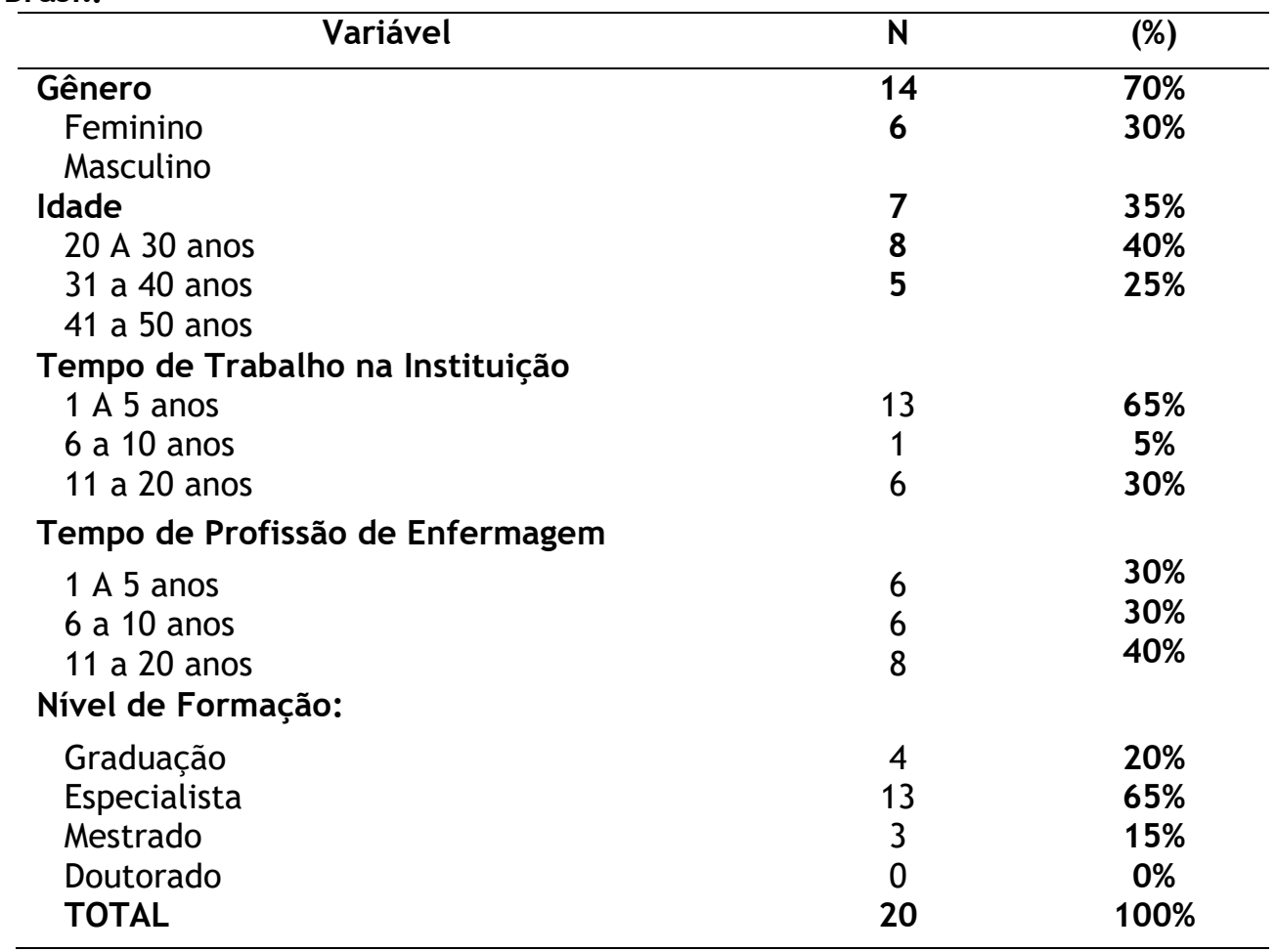

Conhecimentos dos enfermeiros sobre TCE

A Tabela 2 apresenta 0 conhecimento dos profissionais em relação ao TCE. A maioria dos enfermeiros $(90 \%)$ já trabalharam com vítimas de TCE, $95 \%$ relatam sentir-se preparados para assistir as vítimas de TCE, $60 \%$ destacam que existem barreiras importantes que dificultam o atendimento, entre elas, estrutura física e recursos humanos insuficiente. Quanto ao conhecimento sobre a classificação do TCE ocorreu confusão entre o Leve e grave. 
Tabela 2 - Conhecimento dos enfermeiros sobre TCE. Novembro a dezembro de 2019. Região Norte de Mato Grosso, Brasil. $(n=20)$

\begin{tabular}{lccc}
\hline & $\mathbf{n}$ & $\mathbf{( \% )}$ & IVC \\
\hline Trabalha ou já trabalhou com pacientes vítimas de TCE & 18 & $90 \%$ & 0,90 \\
a. Sim & 02 & $10 \%$ & 0,10 \\
b. Não & 00 & $00 \%$ & 0,00 \\
c. Não responderam & & & \\
Se sente preparado para assistir vítimas de TCE & 19 & $95 \%$ & 0,95 \\
a. Sim & 01 & $05 \%$ & 0,05 \\
b. Não & 00 & $00 \%$ & 0,00 \\
c. Não responderam &
\end{tabular}

Existe alguma barreira considerada importante, que dificulte assistir esses pacientes
a. Sim
$12 \quad 60 \% \quad 0,40$
b. Não
$08 \quad 40 \% \quad 0,60$
c. Não responderam

$00 \quad 00 \% \quad 0,00$

Quais são as principais barreiras encontradas na assistência ao paciente com TCE
a. Equipe de enfermagem incapacitada

$\begin{array}{lll}04 & 20 \% & 0,20 \\ 08 & 40 \% & 0,40\end{array}$
b. Estrutura física inadequada e falta de recursos
$08 \quad 40 \% \quad 0,40$

Onde receberam preparo para assistir as vítimas de TCE
a. Prática cotidiana
$08 \quad 40 \% \quad 0,40$
b. Graduação
$06 \quad 30 \% \quad 0,30$
c. Cursos específicos
$06 \quad 30 \% \quad 0,30$

Relação de acertos do Score- TCE leve
d. Sim
$14 \quad 70 \% \quad 1,00$
e. Não
$06 \quad 30 \% \quad 0,00$
a. Não responderam

$00 \quad 00 \% \quad 0,00$

Relação de acertos do Score-TCE moderado
a. Sim
$19 \quad 95 \% \quad 0,75$
b. Não
$01 \quad 05 \% \quad 0,25$
c. Não responderam
$00 \quad 00 \% \quad 0,00$

Relação de acertos do Score- TCE grave
a. Sim
$15 \quad 75 \% \quad 0,95$
b. Não
$05 \quad 25 \% \quad 0,05$
c. Não responderam
$00 \quad 00 \% \quad 0,00$

\section{Conhecimentos dos enfermeiros sobre} a ECG

Todos os enfermeiros (100\%) declaram conhecer a ECG. E constata-se que a maioria $(75 \%)$ utiliza a escala no seu ambiente de trabalho (Figura 1). 
Figura 1 - Conhecimento dos enfermeiros sobre a ECG e avaliação pupilar. Novembro e dezembro de 2019. Região Norte de Mato Grosso, Brasil. $(n=20)$

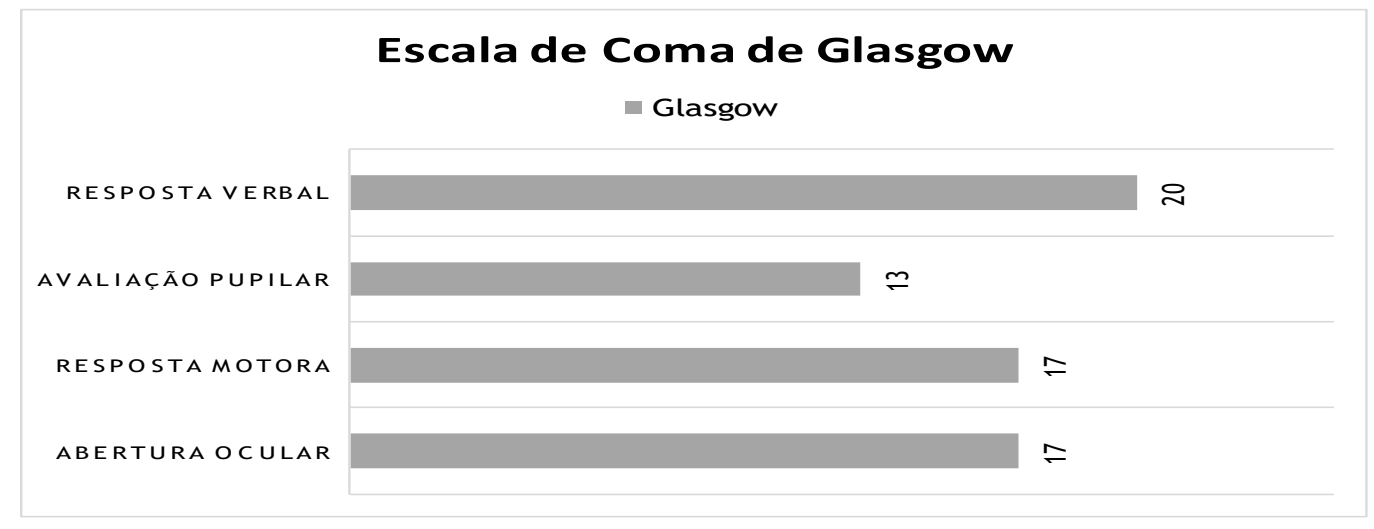

\section{DISCUSSÃO}

Em relação ao sexo, constatou-se que $70 \%$ dos respondentes eram do sexo feminino. Mesmo com o passar dos anos e séculos, por tradição e cultura, a profissão de enfermagem é predominantemente feminina desde a época de Florence Nightingale, no entanto, percebe-se que nos últimos anos, houve um aumento do contingente masculino na enfermagem brasileira ${ }^{12,13}$.

Em relação a faixa etária, tempo de profissão e atuação na instituição, pode-se afirmar que é um público jovem, com potencial de crescimento e amadurecimento profissional. Estão em fase de pleno desenvolvimento, circunstância que colabora para agregação e avanço das práticas profissionais $^{14}$.

Uma pesquisa realizada em um hospital escola público de Goiás (BR), com 63 enfermeiros, constatou que a maioria era do gênero feminino, com idade média de 38,5 anos e especialistas ${ }^{15}$. Outro estudo realizado com uma equipe de enfermagem de um hospital público da região do Médio Araguaia (MT), corrobora com esse predomínio em relação ao gênero, faixa etária e tempo de formação ${ }^{16}$.

Em relação aos conhecimentos sobre TCE, a maioria dos enfermeiros declararam preparados para esse tipo de atendimento (95\%). Apesar disso, 60\% relatam encontrar barreiras importantes durante o atendimento, como falta de capacidade técnica e recurso tecnológico em específico. Isto em si é um fator de grande preocupação para o atendimento de pacientes que exigem atenção extra, vigilância e rigor técnico-científico do enfermeiro ${ }^{17,18}$.

Pesquisa semelhante realizado em Afogados de Ingazeira (PE), destacou 
que entre as principais dificuldades encontradas pelos enfermeiros no atendimento às vítimas de TCE é a falta de equipamentos, e que gera prejuízos para a assistência de enfermagem ${ }^{19}$. No Rio Grande do Norte $(B R)^{11}$ as dificuldades mais encontradas no setor de urgência e emergência são problemas de ordem estrutura física e recursos humanos não habilitados, achados próximos ao presente estudo.

Nota-se que os enfermeiros adquiriram conhecimento para assistência à vítima de TCE no próprio ambiente laboral. É consenso que cursos de atualização, treinamentos e capacitações propiciam melhor dinâmica aos enfermeiros, juntamente com sua equipe. No entanto, é importante que durante a formação de enfermagem, este futuro enfermeiro esteja engajado em ligas acadêmicas e que sejam despertados para conhecimentos prévios sobre TCE tanto em sala de aula como em experiências em campos de estágio Erro! Fonte de referência não encontrada.

A maioria dos participantes foram assertivos em suas respostas principalmente em relação ao TCE moderado, porém, destaca-se o decréscimo de acertos em relação ao TCE leve e o grave. Apesar do número expressivo de acertos (média geral), é necessário intervenção imediata nesse cenário de estudo, que pode ser iniciado com implantação do serviço de educação permanente, sem distanciar das necessidades do profissional que pode não ser apenas de cunho teórico e prático, mas de caráter cognitivo, psicodinâmico e/ou emocional ${ }^{19}$.

Estudo semelhante, com 17 enfermeiros de um hospital de urgência do Estado de Sergipe, destaca acertos para o escore do trauma grave $(80,3 \%)$ e trauma moderado $(81,1 \%)^{20}$. Já em um hospital universitário do Estado de São Paulo, houve uma média de $86 \%$ de acertos entre 127 participantes. Ambas investigações, ratificam os achados desse estudo ${ }^{21}$.

Entre os enfermeiros participantes, $100 \%$ foram assertivos em relação ao item Resposta Verbal da ECG. A média de acertos em relação ao item Resposta Motora e Abertura ocular foram de $85 \%$ para ambos, porém no item avaliação pupilar há uma queda considerável de acertos permanecendo em apenas 65\%, importante pois quanto pior a avaliação pupilar, maior o índice de mortalidade de pacientes com TCE. Estudo recente demonstrou que a reatividade pupilar e o componente motor da ECG apresentaram melhor 
desempenho em prever a morte de vítimas com TCE ${ }^{18}$.

A falta de acurácia dos profissionais para avaliação da ECG pode levar a interpretações errôneas. E para transpor esse risco, o manuseio, o resgaste dos conceitos e a apropriação da escala deve tornar uma realidade no serviço de saúde, a fim de garantir confiabilidade e precisão ${ }^{22,20}$. A utilização de tecnologias digitais é também um importante recurso no contexto do cuidado de enfermagem atual, principalmente na adesão à aplicativos para avaliação de ECG, pois além de fornecer subsídios para 0 profissional na chegada da vítima com TCE à unidade hospitalar, proporciona possibilidade de aprendizado em outros momentos e locais $^{23}$, de forma individual ou coletiva.

Apesar do presente estudo não ter avaliado particularidades da organização do serviço de urgência e emergência nesses atendimentos, pesquisa em um Pronto Socorro Adulto no Rio Grande do Sul (BR) identificou que a assistência da enfermagem não é sistematizada, e muitos enfermeiros avaliam se o paciente está consciente ou inconsciente, mas não utilizam parâmetros como a ECG para evidenciar o nível de consciência, assim em muitas ocasiões o serviço ocorre de forma aleatória e instintiva ${ }^{24}$.

Esse panorama revela que oferecer um cuidado baseado nas tecnologias disponíveis, com equipamentos e espaço físico apropriado, combinado a avaliação criteriosa das necessidades dos pacientes e da equipe de saúde, ainda é um grande desafio diário nas unidades de urgência e emergência em muitos contextos assistenciais do Brasil ${ }^{25}$.

Reconhece que entre as limitações do estudo, destaca-se o fato de ter sido desenvolvida em um único município, com características locais que restringem os resultados à regionalidade. E por ter recrutado somente enfermeiros atuantes nos setores de urgência $e$ emergência, não avaliando o processo de internação do paciente. No entanto, os resultados encontrados poderão servir de alerta aos enfermeiros, e ainda serem utilizados nas unidades hospitalares, para fomentar a capacitação e habilitação profissional no atendimento às vítimas de TCE.

\section{CONCLUSÃO}

Os enfermeiros apresentam conhecimento sobre a definição de TCE e relatam estarem preparados para o 
atendimento, porém, ficou evidenciado algumas barreiras importantes no atendimento. Além disso, percebe-se fragilidades no conhecimento da classificação do TCE leve e grave. Os enfermeiros encontraram dificuldades para os itens da Escala de Coma de Glasgow, sendo o item "avaliação das pupilas" o menos satisfatório.

A identificação dos conhecimentos dos enfermeiros na abordagem às vítimas de TCE auxilia na tomada de decisão e condutas mais seguras. A literatura nacional ainda apresenta poucas publicações sobre a temática, principalmente na associação com a qualidade da assistência de enfermagem. Dessa forma, é fundamental que estudos mais amplos sejam realizados e que possuam 0 objetivo de contribuir com a realidade encontrada.

\section{REFERÊNCIAS}

1. Munivenkatappa A, Agrawal A, Shukla DP, Kumaraswamy D, Devi Bl. Traumatic brain injury: does gender influence results? Int J CritlllnInj Sci. 2016; 6(2):70-3.

2. Peeters W, Brande RVD, Polinder S, Brazinova A, Steyberg EW, Lingsma $\mathrm{HF}$, Maas AIR. Epidemiology of traumatic brain injury in Europe. Acta neurochir. 2015; 157(10):1683-96.

3. Marinho CSR, Santos JNA, Morais Filho LA, Valença CN, Santos EGO, Bay Júnior OG. Acidente de trânsito: análise dos casos de traumatismo cranioencefálico. Enferm Glob. 2019; 54:343-52.

4. Salazar LRM, Rubiano AM, Miranda HRA, Miranda WC, Cerra GA, Rivera $M A B$, et al. Severe traumatic brain injury: pre-hospital care, surgical treatment and multimodal monitoring. Bull Emerg Trauma. 2016; $4(1): 8-23$.

5. Sousa RM, Fernandes L, Graça EP, Gomes OV. Epidemiologia de traumatismo craniencefálico em um hospital. Rev Soc Bras Clín Méd. 2018; 16(1): 21-24.

6. Silva ZA, Pio TM, Maia LFS. Trauma cranioencefálico: intervenções do enfermeiro no atendimento préhospitalar. Rev Recien. 2019; 9(27):46-53.

7. Santos JEB, Costa JBC, Arcanjo HS, Pinto JR. Accidents with motorcycles: a view of nurses of the urgent mobile care services. Rev Pesqui (Univ Fed Estado Rio J Online). 2020; 12(1):400405.

8. Santos MAS, Santos LGE, Oliveira GFSM, Miranda LM. Assistência de 
enfermagem ao paciente politraumatizado. Cad Grad Ciênc Hum Soc Unit. 2018; 4(02):11-22.

9. Freitas FFB, Mattos GSS, Cavalcante AC, Pinheiro MBGN, Sousa MNA. Papel do enfermeiro no serviço de acolhimento e classificação de risco no setor de urgência e emergência. Rev Interdisciplin Saúde. 2015; 2(3):314-333.

10. Camargo DR, Luz L, Aquino ROS, Gomes RMJ, Oliveira ZG. A comunicação na enfermagem como estratégia de liderança na assistência. Enferm Brasil. 2011; 10(5):309-313.

11. Boratelli FR. Conhecimento dos enfermeiros sobre 0 processo de cuidar do paciente com traumatismo cranioencefálico [dissertação]. Natal: Universidade Federal do Rio Grande do Norte; 2010, p.181.

12. Machado MH, Aguiar Filho W, Lacerda WF, Oliveira E, Lemos W, Wermelinger $M$, et al. Características gerais da enfermagem: o perfil sócio demográfico. Enferm foco. 2016; 7(esp):9-14.

13. Conselho Federal de Enfermagem. Pesquisa perfil da enfermagem no Brasil. 2015. [Acesso em 08 de junho de 2020]. Disponível em: http://www.cofen.gov.br/perfilenfe rmagem/index.html.

14. Quaresma AS, Xavier DM, Cezar-Vaz MR. O papel do enfermeiro na classificação de risco nos serviços de urgência e emergência. Rev Enferm Atual InDerme. 2019; 87(esp):2-10.

15. Sousa TV, Melchior LMR, Bezerra MLR, Carvalho Filha FSS, Santos OP, Pereira MC, et al. Conhecimento de enfermeiros sobre sepse e choque séptico em um hospital escola. J Health NPEPS. 2020; 5(1):132-146.

16. Liba YHAO, Lemes AG, Oliveira PR, Nascimento VF, Fonseca PIMN, Volpato RJ, et al. Percepções dos profissionais de enfermagem sobre 0 paciente pós-tentativa de suicídio. J Health NPEPS. 2016; 1(1):109-121.

17. Varghese R, Chakrabarty J, Menon G. Nursing management in adults with severe traumatic brain injury: a narrative review. Indian J Crit Care Med. 2017; 21(10):684-697.

18. Oyesanya TO, Brown RL, Turkstra LS. Caring for patients with traumatic brain injury: a survey of nurses' perceptions. J Clin Nurs. 2017; 26(11-12):1562-1574.

19. Soares CB, Veras D, Siqueira JD, Martins ENX, Sousa KMO, Alves ÉSRC. Condutas de enfermeiros ao paciente vítima de traumatismo 
cranioencefálico. Temas saúde. 2017; 17(1):81-103.

20. Oliveira DMP, Pereira CU, Freitas ZMP. Conhecimento do enfermeiro sobre avaliação neurológica do paciente com trauma cranioencefálico. Rev enferm UFPE on line. 2016; 10 (supl. 5):42494254.

21. Santos WC, Vancini-Campanharo CR, Lopes MCBT, Okuno MFP, Batista REA. Avaliação do conhecimento de enfermeiros sobre a escala de coma de Glasgow em um hospital universitário. Einstein (São Paulo). 2016; 14(2):213-218.

22. Mattar I, Liaw SY, Chan MF. Nurses' self-confidence and attitudes in using the Glasgow Coma Scale: a primary study. Nurs Crit Care. 2015; 20(2):98-107.
23. Barros WCTS, Dal Sasso GTM, Alvarez AG, Ramos SF, Martins SR. Aplicativo para avaliação do nível de consciência em adultos: produção tecnológica em enfermagem. Cogitare enferm. 2019; 24:e60338.

24. Werlanga SL, Badkebc MR, Freitaga VL, Silva GS, Federizzie DS, Ribeiro MV. Enfermagem na Assistência ao Traumatismo Cranioencefálico em um Hospital Universitário. J Health Sci. 2017; 19(2):177-82.

25. Pereira KC, Ferreira WFS. Classificação de riscos no atendimento de urgência $e$ emergência: contribuição do enfermeiro. Rev jurid Uniandrade. 2020; 31(1):1-12.

Financiamento: Os autores declaram que não houve financiamento.

Conflito de interesses: Os autores declaram não haver conflito de interesses.

Participação dos autores:

- Concepção: Rezer F, Pereira BFO, Faustino WR.

- Desenvolvimento: Rezer F, Pereira BFO, Faustino WR.

- Redação e revisão: Rezer F, Pereira BFO, Faustino WR.

Como citar este artigo: Rezer F, Pereira BFO, Faustino WR. Conhecimento de enfermeiros na abordagem à vítima de traumatismo cranioencefálico. J Health NPEPS. 2020; 5(2):291-302. 